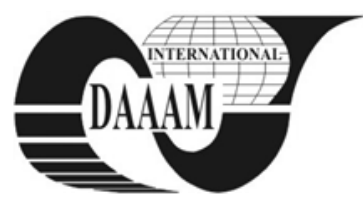

Annals of DAAAM for 2011 \& Proceedings of the 22nd International DAAAM Symposium, Volume 22, No. 1, ISSN 1726-9679 ISBN 978-3-901509-83-4, Editor B. Katalinic, Published by DAAAM International, Vienna, Austria, EU, 2011 Make Harmony between Technology and Nature, and Your Mind will Fly Free as a Bird Annals \& Proceedings of DAAAM International 2011

\title{
MICROCONTROLLER BASED BIOSIGNAL ACQUISITION AND ANALYSIS SYSTEM
}

\author{
SZAKACS SIMON, P[eter] \& MORARU, S[orin] - A[urel]
}

\begin{abstract}
This paper presents a microcontroller-based project for acquisition of the EKG and EOG signals and transfers to the computer for recording and data analysis. The main purpose of the project is to record both type of signals using the same multichannel device, the instrumentation amplifier and the power amplifier of every channel supports adjustable gain, so can be calibrated. The importance of calibration is due to difference in voltage of the EKG and EOG signals and because every person has different gain of those signals. Analog signal is converted in digital with Atmel microcontroller and transferred to the computer trough serial interface. We developed a PC based software for data recording and signal analysis, resulting the values of the heart rate and the direction of the eye movement
\end{abstract}

Key words: biosignal, microcontroller, data acquisition, EKG, EOG

\section{INTRODUCTION}

Biosignal analysis is part of the modern medicine, is noninvasive, used for detect a wide range of disease (heart, muscle, or neurological disorders). Eye activity is suitable to drive human interface devices like computer mouse, joystick or remote controller.

The EKG signal is one of the simplest procedures to evaluate the heart, based on its electrical activity recording. Electrodes are placed at certain locations on the arms, legs and chest. The resulting signal is differential type with respect to the virtual ground electrode (e.g. right leg). (Dale D. 2008)

The eyes are the origin of a steady electric potential field, which can also be detected in total darkness and if the eyes are closed. It is generated by a dipole with its positive pole at the cornea and its negative pole at the retina. The magnitude of this so-called corneo-retinal potential difference (CRP) lies in the range of $0.4 \mathrm{mV}$ to $1.0 \mathrm{mV}$. On the assumption of an unchanging CRP, the electric signal that can be derived using two pairs of skin electrodes placed at periorbital positions around one eye is called Electrooculogram (EOG). EOG typically shows signal amplitudes ranging from $5 \mu \mathrm{V}$ to $20 \mu \mathrm{V}$ and essential frequency content between $0 \mathrm{~Hz}$ and $30 \mathrm{~Hz}$. (Bulling A. et al. 2009)

For developing biosignal-based applications, we need a data acquisition system at low cost, suitable for EKG and EOG recordings. Existing applications use one of the signals, for our needs, we developed a multichannel hardware with adjustable gain and PC based software for data analysis. EKG cable is very expensive; we made it from quality shielded audio cable. The quality of the signal is good, due to digital noise filter, shielded cable, and low noise instrumentation amplifier and low noise power amplifier we used. The system we developed implements the following functions: EKG recording, calculating heart rate, detecting eye movements left, right, up, down, blinking. In the next step, we purpose to extend the numbers of EKG channels, detailed automatic waveform analysis, and development of human interface devices.

\section{EXISTING HARDWARE PARTS}

After studying the market about existing hardware resources like electrodes, cables, instrumental amplifiers, power amplifiers, filters and microcontrollers, we decided for Atmega 8 microcontroller, AD620A instrumentation amplifier, MAX 7426 fifth order low-pass digital filter.

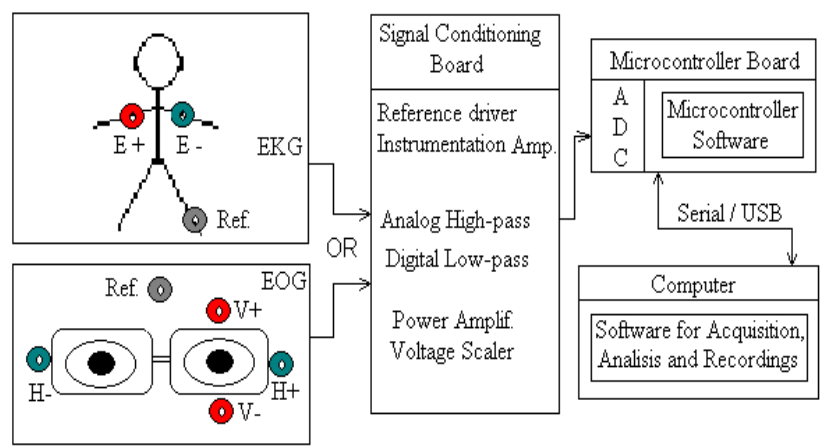

Fig. 1. Block diagram of the system

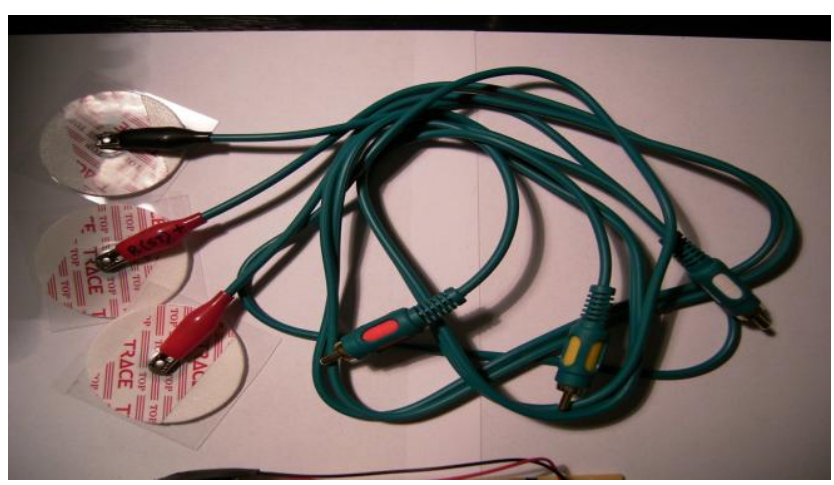

Fig. 2. EKG electrodes and shielded cable

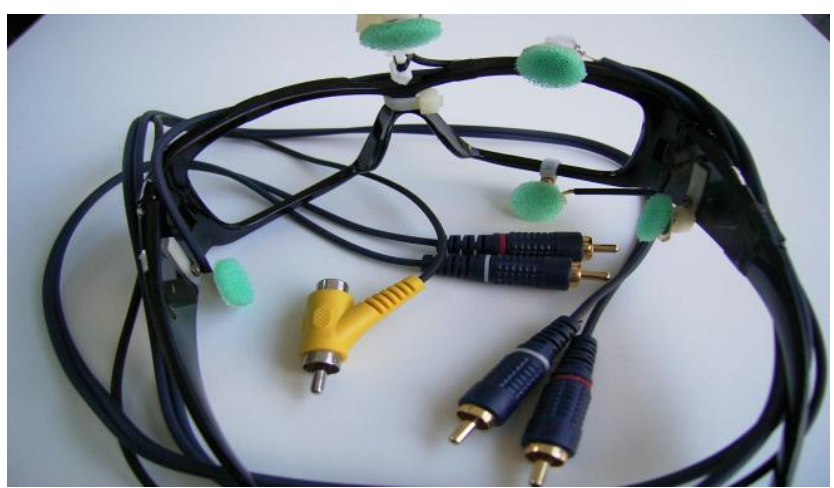

Fig. 3. EOG Goggles and shielded cable 


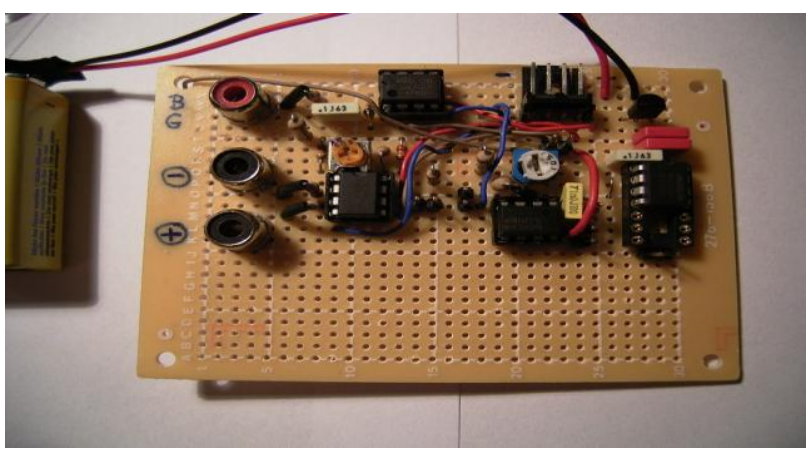

Fig. 4. Signal conditioning board

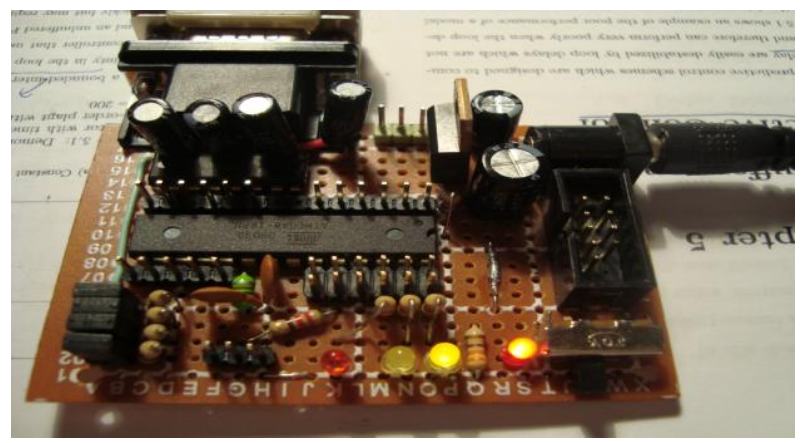

Fig. 5. Microcontroller board

The first stage of our design is the electrodes. The electrodes were chosen with the concern of protecting the eyes from hazardous elements. ECG disposable electrodes were used because of their easy availability. Silver/Silver-Chloride electrodes were chosen because the half-cell potential was the closest to zero. (Vinodh R. et al. 2008)

On Figure 1 we present the functional block diagram of the system, it can receive the EKG or EOG signals, which are processed, displayed and recorded. Figure 2 and Figure 3 presents the input hardware, we used the same type of $\mathrm{AgCl}$ electrodes for both biosignals. Signal conditioning board presented on Figure 4 includes the following: reference driver for virtual ground electrode, instrumentation amplifier for quality signal acquisition, analog high pass filter and digital low pass filter for noise filtering, power amplifier and voltage scaler to adjust input for the ADC.

Figure 5 presents the microcontroller board with serial interface.

\section{SOFTWARE RESOURCES}

This project includes two software parts, the first is developed to drive the microcontroller $(* * * 2010)$ and the second for presenting the results on the computer.

To develop the microcontroller software, we used AVR Studio development environment and PoniProg memory programming software. (*** 2005)

The PC based software was developed in Lab Windows CVI environment and displays the results of the acquisition and analisis. This software contains the following functions: serial port initialization, EKG signal acquisition, Acquisition reset, EKG waveform display, Heart rate calculation, heart rate abnormalities detection, EOG signal detection for left, right, up, down movements and blinking.

For PC based software we used multi threading technology, one tread is for data acquisition and the others are for analisis and recordings. We used this programming method because in one thread version, all the interface buttons are inactive until the data acquisition completes.

\section{CONCLUSION}

The system designed for biosignal acquisition can record the EKG and EOG signals, the waveforms are displayed on the screen of a computer, the software calculates the heart rate of the patient and detects the presence and type of EOG signals. This design with adjustable gain is more economical compared to separate amplification and filter circuits.

The high frequency noise was successful rejected due to fifth order digital low-pass filter. The cost requirements are met, and the system can be used to develop other biosignal-based projects.

We used hardware filtering because software filtering it will significantly affect on the real-time nature of the system, as the software like MATLAB takes heavy time to process and filter the digital data. (Malik A. et al. 2007)

Today, it is not so difficult to construct such a device, but there are many standards concerned to human safety especially for intensive health care usage. (Petrov G. 2004)

\section{FUTURE DIRECTIONS}

The next step of our research is to minimize the size of the heart monitor, add wireless module, and use it for assisted living project. A medical-oriented sensor network system for assisted living facilities, integrates heterogeneous devices, some wearable on the patient and some placed inside the living space. Together they inform the healthcare provider about the health status of the resident. Data is collected, aggregated, preprocessed, stored, and acted upon using a variety of replaceable sensors and devices (activity sensors, physiological sensors and environmental sensors). (Stankovic, J. A., 2006)

In the assisted living project, we intend to use EOG based interactions to control the environment status and home appliances. An advantage is that EOG processing requires less computational power than video due to lower data rates. This enables an embedded and low-power design and results in low data storage. (Bulling A. et al. 2009)

\section{ACKNOWLEDGEMENTS}

This paper is supported by the Sectoral Operational Programme Human Resources Development, financed from the European Social Fund and by the Romanian Government under the project number POSDRU/107/1.5/S/76945.

\section{REFERENCES}

Dale, D., (2008) Rapid Interpretation of EKG`s, 6-th edition, Medical edition, Bucuresti, ISBN: 978-973-339-0647-6

Bulling, A., Roggen, D., Troster G., (2009) Wearable EOG goggles: Seamless sensing and context-awareness in everyday environments, ETH, Wearable Computing Laboratory, Zurich

Stankovic, J. A., (2006), Wireless Sensor Networks, Department of Computer Science, University of Virginia Charlottesville, Virginia 22904

Malik. A. Q., Ahmad, J., (2007), Retina based mouse control, World Academy of Science, Engineering and Technology, 31, 2007.

Petrov, G. (2004), www.codeproject.com, ECG recording, storing, filtering and recognition

*** (2010) http://ham.elcom.pub.ro/, RC microcontroler.PDF, Aspects of using the AVR microcontrollers resources, M.S.

*** (2005), AVR simulation with the ATMEL AVR Studio 4, Purdue University, Rev.C. 